Turkish

Journal of

Nephrology

\title{
Lupus Nephritis Presenting with Preeclampsia
}

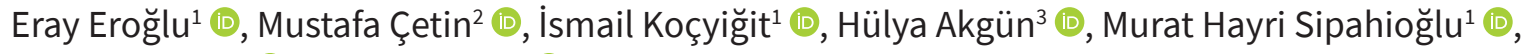 \\ Bülent Tokgöz ${ }^{1}$ (D), Oktay Oymak ${ }^{1}$ (i) \\ ${ }^{1}$ Division of Nephrology, Erciyes Üniversitesi School of Medicine, Kayseri, Turkey \\ ${ }^{2}$ Department of Internal Medicine, Erciyes Üniversitesi School of Medicine, Kayseri, Turkey \\ ${ }^{3}$ Department of Pathology, Erciyes Üniversitesi School of Medicine, Kayseri, Turkey
}

\begin{abstract}
Systemic lupus erythematosus (SLE) is an autoimmune disease characterized by multisystem involvement. The renal involvement of SLE may present with hematuria , proteinuria or acute kidney dysfunction. It is well established that pregnancy may trigger the disease activation in patients with SLE. Lupus nephritis $(L N)$ may be diagnosed during pregnancy in very few patients. Preeclampsia is a pregnancy-related disorder characterized by maternal hypertension, proteinuria, and edema and is sometimes complicated by renal dysfunction, which usually occurs in the last trimester of pregnancy. However preeclampsia rarely occurs within $48 \mathrm{~h}$ of postpartum. In this report, we present the case of a 20 -year-old patient with oliguria, proteinuria, edema, and hypertension who was diagnosed with preeclampsia starting at the 35th week of her first pregnancy. Acute kidney failure developed in the postpartum period after emergency cesarian delivery. Crescentic and diffuse LN was revealed by renal biopsy. While SLE is a risk factor for preeclampsia, LN should be considered in kidney failure in the third trimester or postpartum period. Keywords: Pregnancy, preeclampsia, lupus nephritis
\end{abstract}

Corresponding Author: Eray Eroğlu $\bowtie$ drerayeroglu@hotmail.com

Received: 16.09.2018 Accepted: 30.09.2018

Cite this article as: Eroğlu E, Çetin M, Koçyiğit I, Akgün H, Sipahioğlu MH, Tokgöz B, et al. Lupus Nephritis Presenting with Preeclampsia. Turk J Nephrol 2020; 29(1): 84-8.

\section{INTRODUCTION}

Systemic lupus erythematosus (SLE) is an autoimmune disease with multisystem involvement. Approximately $50 \%$ of patients with SLE may present with renal involvement. Renal involvement typically manifests as hematuria, proteinuria, or sudden onset renal dysfunction. In 1957, Muehrcke et al. (1) described lupus nephritis (LN) by kidney biopsy for the first time. $L N$ is a serious but curable disease. Clinically, LN may also present with nephrotic syndrome, nephritic syndrome, or crescentic glomerulonephritis. Clinicopathologically, LN is classified into six types. The most frequent and the most severe prognosis is the form with diffuse proliferative glomerulonephritis (type IV) (2). Approximately $15 \%-25 \%$ of patients progress to end-stage renal disease within 10 years.

Several studies have demonstrated that pregnancy leads to the activation of this disease in women with SLE (3-6). Chronic LN, if activated during pregnancy, can lead to hypertension and renal failure, which can adversely affect the health of the mother and fetus. In rare cases, LN can be diagnosed during pregnancy. In particular, the clinical findings of patients who have not been previously diagnosed with LN may become apparent following activation during pregnancy. However, it is possible that the physiological changes related to pregnancy may mask some of the clinical presentations of LN including of proteinuria and edema. Otherwise, various diseases may cause renal dysfunction during pregnancy $(4,5)$. Among these, there are many possible causes of acute renal failure in pregnancy such as preeclampsia, hemolytic uremic syndrome, and perioperative complications (acute tubular necrosis) and rapidly progressive glomerulonephritis $(2,6)$. Preeclampsia is an important health problem that causes serious maternal and fetal complications in approximately $7 \%-10 \%$ of all pregnancies, mostly during the last trimester and rarely within $48 \mathrm{~h}$ postpartum. It is characterized by systemic vaso- 


\begin{tabular}{|c|c|c|}
\hline Parameters & Value & Normal range \\
\hline White blood cell $\left(10^{3} / \mu \mathrm{L}\right)$ & 7.1 & $4.8-10.7$ \\
\hline Hemoglobin (g/dL) & 8.9 & $12-16$ \\
\hline Platelets $\left(10^{3} / \mu \mathrm{L}\right)$ & 204 & $130-400$ \\
\hline Glucose (mg/dL) & 110 & $74-106$ \\
\hline BUN (mg/dL) & 32 & $8-23$ \\
\hline Creatinine (mg/dL) & 1.82 & $0.7-1.20$ \\
\hline Uric acid (mg/dL) & 8.1 & $2.4-5.7$ \\
\hline Calcium (mg/dL) & 8.7 & $8.6-10.2$ \\
\hline Phosphorus (mg/dL) & 6.5 & $2.5-4.5$ \\
\hline Magnesium (mmol/L) & 1.2 & $0.66-1.77$ \\
\hline Sodium (mmol/L) & 136 & $136-145$ \\
\hline Potassium (mmol/L) & 6.03 & $3.5-5.1$ \\
\hline Clor (mmol/L) & 103 & $98-107$ \\
\hline Total bilirubin (mg/dL) & 0.15 & $0.2-1.2$ \\
\hline Direct bilirubin (mg/dL) & 0.09 & $0-0.3$ \\
\hline GGT (U/L) & 11 & $10-71$ \\
\hline $\mathrm{LDH}(\mathrm{U} / \mathrm{L})$ & 411 & $135-225$ \\
\hline AST (U/L) & 39 & $0-40$ \\
\hline ALT (U/L) & 28 & $0-41$ \\
\hline $\operatorname{ALP}(U / L)$ & 151 & $40-130$ \\
\hline Total protein (g/dL) & 4.3 & $6.4-8.3$ \\
\hline Albumin (g/dL) & 2.1 & $3.5-5.2$ \\
\hline PT (s) & 10 & $10.1-14.9$ \\
\hline APTT (s) & 26 & $20-36$ \\
\hline INR & 0.89 & $0.8-1.2$ \\
\hline \multicolumn{3}{|l|}{ UDT } \\
\hline Protein (mg/dL) & 100 & $0-10$ \\
\hline Blood (ery/ $/ \mathrm{L})$ & 80 & $0-5$ \\
\hline Leu (leu/ $\mu \mathrm{L})$ & 29 & $0-10$ \\
\hline \multicolumn{3}{|l|}{ Urine microscopy } \\
\hline Erythrocyte (HPF) & 80 & $0-3$ \\
\hline Leukocyte (HPF) & 29 & $0-5$ \\
\hline Urine microprotein (mg/dL) & 2950 & $28-217$ \\
\hline Urine creatinine (mg/dL) & 289 & $0-15$ \\
\hline \multicolumn{3}{|c|}{$\begin{array}{l}\text { BUN: blood urea nitrogen; GGT: gamma-glutamyl transferase; LDH: lactate dehy- } \\
\text { drogenase; AST: aspartate aminotransferase; ALT: alanine aminotransferase; ALP: } \\
\text { allkaline phosphatase; PT: prothrombin time; APT: activated partial thromboplas } \\
\text { tin time; INR: international normalized ratio; UDT: Urine dipstick test }\end{array}$} \\
\hline
\end{tabular}

spasm, maternal hypertension, proteinuria, edema, increased platelet aggregation, and decreased uteroplacental blood flow and is observed in the presence of placental tissue and resolves within a short time after birth. The pathophysiology of the disease are still unclear (7-9).

In this case report, we describe the case of a 20-year-old patient without a history of any disease who developed proteinuria, edema, and hypertension at 35 weeks of her first pregnancy. She underwent emergency cesarean section (C/S) due to preeclampsia, developed oliguria and renal dysfunction in the postpartum period, and was referred to our clinic. Subsequently, she was diagnosed with crescentic LN with renal biopsy.

\section{CASE PRESENTATION}

A 20-year-old woman without a history of kidney disease presented to a private hospital with elevated blood pressure and swelling of the lower limbs during 35th week of her first pregnancy. Emergency C/S was performed due to the diagnosis of preeclampsia. Patient's postoperative urine output decreased ( $200 \mathrm{cc}$ in $12 \mathrm{~h}$ ) and serum creatinine level increased to $1.8 \mathrm{mg} /$ $\mathrm{dL}$; so urinary system ultrasound was performed and ectasia was not detected in the pelvicalyceal system. Kidney sizes were reported within normal range. In the postoperative period, 40 mg furosemide was administered intravenously (iv) due to oliguria. She was transferred to the nephrology department of our university hospital in April 2017 with the preliminary diagnosis of oliguric acute renal failure. There were no complications such as perioperative bleeding, hypovolemia, and hypotension. There was no history of nonsteroidal anti-inflammatory drug use. Complaints of fatigue and oliguria were noted in the systemic query. On physical examination, her body temperature was $37.0^{\circ} \mathrm{C}$, pulse rate was 112 beats $/ \mathrm{min}$, blood pressure was $130 / 80 \mathrm{mmHg}$, and respiratory rate was 22 breaths/min; decreased breathing sounds in the lower lung fields at the bottom of the rib cage was detected by auscultation and significant amount of ascites was detected in the abdomen and pretibial +++/+++ edema was detected in the examination of distal extremities. The laboratory test results are shown in Table 1.

Ten grams of proteinuria was detected in the spot urine microprotein/creatinine test. Microscopic examination of urinary sediment revealed dysmorphic erythrocytes, leukocyte casts , and granular casts. Urinary ultrasonography revealed that both kidney sizes were normal, parenchymal echoes increased to grade 1 in both kidneys without pelvicalyceal ectasia and stones, and intra-abdominal ascites was present. The patient's renal function tests deteriorated during the following days in the nephrology clinic. There were no abnormal results in the urine gram, and no bacteria were detected in urine culture. Diuretic treatment was initiated as the patient had increased pretibial edema, oliguric course, and hypervolemia. There was no response to diuretic treatment with furosemide. At the follow-up, the patient developed anuria and diuretic-resistant hypervolemia and was treated with intermittent hemodialysis (HD) and ultrafiltration 
(UF). The patient received intermittent HD+UF treatment in the following days. After dialysis, regression was observed in hypervolemia findings. Urine output remained oliguric. An autoimmune panel was obtained from the patient, who showed signs of proteinuria, hypoalbuminemia, edema, oliguric renal failure, and active urine sediment. Renal biopsy was performed. After the pathological examination of the kidney biopsy revealed crescent formation, the patient was given pulse steroid treatment ( $1 \mathrm{~g} /$ day for 3 days), followed by cyclophosphamide + mesna iv $(750 \mathrm{mg} /$ day) immunosuppressive therapy. Based on the autoimmune panel findings, the patient was diagnosed with SLE with antinuclear antibody (ANA) positivity, anti-double stranded DNA antibody ( anti-dsDNA ) positivity, and lupus anticoagulant positivity, and low levels of complement factor 3 (C3) and complement factor 4 (C4) were noted. The patient was evaluated for other SLE findings. There was no history of oral aphthae or arthralgia. Mild hyperemia was noted on the face. However, typical malar rash was not detected. As a result of the pathology, type IV diffuse $\mathrm{LN}+$ crescentic glomerulonephritis was reported (Figure 1). Hydroxychloroquine treatment was initiated after the ophthalmology consultation. Simultaneous plasmapheresis treatment was planned. The patient underwent plasmapheresis for seven sessions. At the follow-up, her urine output increased after the 6th session of plasmapheresis (Figure 2). Intermittent dialysis was also administered during this period.

The patient's serum blood urea nitrogen (BUN)/creatinine (Cre) level decreased from $61 / 5.7 \mathrm{mg} / \mathrm{dL}$ to $47 / 2.43 \mathrm{mg} / \mathrm{dL}$. She was

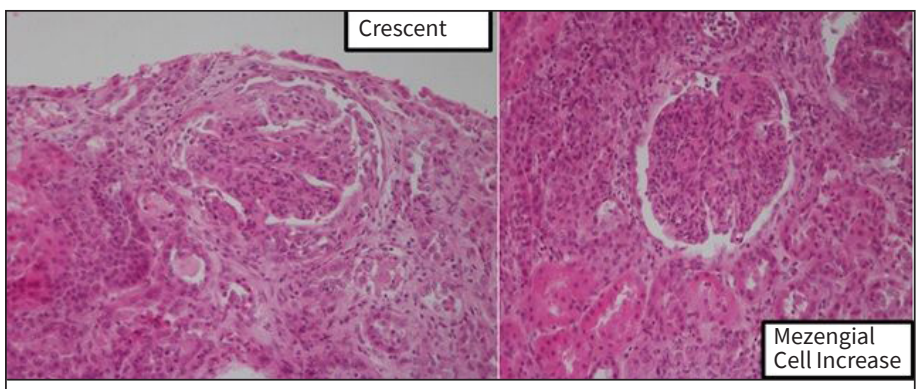

Figure 1. Presence of crescent formation and mesangial cell increase in glomeruli in renal biopsy specimen (H\&E, $\times 40)$. discharged with a twice weekly intermittent HD program and monthly cyclophosphamide treatment plan. She received six cycles of cyclophosphamide treatment. Dialysis treatment was discontinued in July 2017 when her renal function improved. In November 2017, BUN/Cre level of $6.2 / 0.48 \mathrm{mg} / \mathrm{dL}$ and proteinuria of $6.7 \mathrm{~g} /$ day were noted. Maintenance therapy of mycophenolate mofetil was started, and out-patient clinic controls were continued in remission.

\section{DISCUSSION}

Systemic lupus erythematosus is the most common rheumatologic disease in pregnancy because it is usually observed in women in the reproductive age group. Fertility in women with SLE is similar to that in the normal population. The exacerbation of the disease during pregnancy and in the postpartum period is approximately $50 \%$. In patients with lupus and pregnancy, the disease is usually exacerbated in the last trimester of pregnancy and within a few weeks after birth. Lupus exacerbations during pregnancy are frequently associated with renal and hematologic systems (1-4). Many complications in lupus can occur during pregnancy. These complications include exacerbation of the disease (leukomotor-hematologic-renal), abortion (especially in the presence of antiphospholipid antibodies), premature birth, intrauterine growth retardation, hypertension, increased risk of preeclampsia (especially in nephritis cases), risk of renal failure development, and maternal death. Therefore, the follow-up of pregnant women with SLE, which can cause significant maternal and fetal complications, should be performed with care. On the other hand, patients who become pregnant before the diagnosis of SLE may admit to the hospital with lupus complications. In the present report, we described a patient diagnosed with preeclampsia and $\mathrm{LN}$ due to oliguric acute renal failure after $\mathrm{C} / \mathrm{S}$.

Preeclampsia is defined as a combination of proteinuria, edema and increased blood pressure as $>140 / 90 \mathrm{mmHg}$ or increase in systolic blood pressure by $30 \mathrm{mmHg}$ and increase in diastolic blood pressure by $15 \mathrm{mmHg}$ in pregnant women at $>20^{\text {th }}$ week pregnancy, and the definitive treatment of preeclampsia is delivery of the fetus. Although preeclampsia and LN may coexist during pregnancy, it is very important to establish the differen-

\begin{tabular}{|c|c|c|c|c|c|c|c|c|c|c|}
\hline & & & Biopsy & Steroid $+C$ & Shosphamide & & heresis & & & \\
\hline Date & 21 April & 25 April & 29 April & 3 May & 6 May & 9 May & 12 May & 15 May & 18 May & 21 May \\
\hline BUN (mg/dL) & 32 & 17 & 13 & 22 & 48 & 47 & 34 & 39 & 53 & 47 \\
\hline Creatinine $(\mathrm{mg} / \mathrm{dL})$ & 1.89 & 2.34 & 2.6 & 5.2 & 5.2 & 5.2 & 3.2 & 3.2 & 2.6 & 2.4 \\
\hline $\begin{array}{l}\text { Received } \\
\text { (volum-cc) }\end{array}$ & 1300 & 2500 & 2200 & 3350 & 1700 & 2600 & 3000 & 4250 & 1350 & 2100 \\
\hline $\begin{array}{l}\text { Removed } \\
\text { (volum-cc) }\end{array}$ & 350 & 150 & 50 & Yok & 50 & 200 & 750 & 4850 & 3250 & 4250 \\
\hline
\end{tabular}

Figure 2. Patient's clinical follow-up and treatment schedule. 
tial diagnosis as their treatments are different. Steroids are used to treat LN, which worsens preeclampsia. Therefore, signs and symptoms that are more reliable in showing SLE exacerbation and diagnosis in pregnancy can be summarized as ANA positivity, increased anti-dsDNA autoantibody titer (if known before), lymphopenia, active urine sediment, erythrocyte casts, direct Coombs test positivity, hemolytic anemia, fever, lymphadenopathy, typical oral mucosal ulcers, inflammatory arthritis, and cutaneous vasculitis. However, findings such as hypertension, edema, proteinuria, impaired renal function, and thrombocytopenia are common factors in both preeclampsia and exacerbation of SLE. In addition, proteinuria that occurs during pregnancy may be at physiological limits or may be related to lupus. In this context, the presence of hematuria and examination of urine sediment may be suggestive of renal involvement due to $\operatorname{SLE}(2,3,8)$. Anti-dsDNA positivity with hypocomplementemia, even in the absence of clinical activity, may be a predictor of abortion or preterm birth, particularly in the second trimester of SLE pregnancies. C3 and C4 levels are typically reduced by $25 \%$ in LN. In contrast, their levels may increase by $10 \%-15 \%$ in pregnancy and preeclampsia.

Performing renal biopsy in $L N$ is crucial in determining the treatment, identifying patients who need urgent treatment, evaluating the response to treatment, and predicting the prognosis (10).

The prognosis is better if SLE is in clinical remission for at least 6 months before pregnancy. The live birth rate is approximately $90 \%$. However, the maternal mortality rate of patients with active disease in the last 6 months before the conception is approximately $15 \%$. Approximately $60 \%$ of infants born from these mothers have morbidity or mortality. If SLE first appeared during pregnancy, maternal and fetal prognoses are similar to those patients with SLE who have active disease in the last 6 months before the conception. Only heart blocks have been reported as congenital problems in infants of mothers with SLE (11). Even in the case series with best results, some patients with LN develop renal failure that requires dialysis. Dialysis support may be discontinued in $10 \%-28 \%$ of these patients. These are usually patients with rapid renal dysfunction, suggesting acute and potentially reversible disease activation. Only $1 \%-4 \%$ of the chronic dialysis and transplant population are patients with lupus. The clinical and serological activities of lupus usually decrease after reaching end-stage renal failure. The majority of deaths in patients with lupus occurs during the first 3 months of dialysis and is usually caused by an infection. After three months mortality causes are cardiovascular disease and infection. The prognosis of patients surviving during the first 3 months of dialysis is not different from that of patients without lupus (12).

Our patient was admitted to the hospital with suddenly elevated blood pressure and swelling of the lower limbs when she was in the $35^{\text {th }}$ week of pregnancy. . Emergency $\mathrm{C} / \mathrm{S}$ was performed with a diagnosis of preeclampsia. Urinary system ultrasound were reported as normal after the patient's urine output decreased (200 mL in $12 \mathrm{~h}$ ) and postoperative serum creatinine increased to $1.8 \mathrm{mg} / \mathrm{dL}$. She was transferred to our nephrology clinic with a preliminary diagnosis of oliguric acute renal failure. She was diagnosed with LN after autoimmune serology and renal biopsy results. The patient underwent dialysis for approximately 1 month, and her renal function improved with immunosuppressive and plasmapheresis therapy.

\section{CONCLUSION}

SLE may be the underlying cause of preeclampsia during pregnancy with concomitant renal dysfunction. Physicians should be aware of SLE as an unusual cause of preeclampsia during pregnancy.

Informed Consent: Written informed consent was received from the patient who participated in this study.

Peer-review: Externally peer-reviewed.

Author Contributions: Concept - E.E.; Design - E.E.; Supervision - I..K.; Resource - M.C..; Materials - M.Ç., H.A.; Data Collection and/or Processing - M.Ç., E.E., H.A.; Analysis and/or Interpretation - E.E., M.Ç.; Literature Search - M.Ç., E.E; Writing - M.Ç., E.E.; Critical Reviews-M.H.S., B.T., O.O.

Conflict of Interest: The authors have no conflict of interest to declare.

Financial Disclosure: The authors declare that this study has received no financial support.

\section{REFERENCES}

1. Muehrcke RC, Kark RM, Pirani CL, Pollak VE. Lupus nephritis: a clinical and pathological study based on renal biopsies. Medicine (Baltimore) 1957; 36: 1-145. [CrossRef]

2. Murrah FA. Lupus erythematosus in pregnancy. J Obstet Gynaecol Br Emp 1958; 65: 401-8. [CrossRef]

3. Garsenstein M, PollakVE, Kark RM. Systemic lupus erythematosus and pregnancy. New Engl J Med 1962; 267: 165-9. [CrossRef]

4. Estes D, Larson DL. Systemic lupus erithematosus and pregnancy. Clin Obstet Gynecol 1965; 8: 307-21. [CrossRef]

5. Bear RA. Pregnancy in patients with renal disease: A study of 44 cases. Obstet Gynecol 1976; 48: 13-26.

6. Houser MT, Fish AJ, Tagats GE, Williams PP, Michael AF. Pregnancy and systemic lupus erythematosus. Am J Obstet Gynecol 1980; 138: 409-13. [CrossRef]

7. Mikhail MS, Anyaegbunam A, Garfinkel D, Palan PR, Basu J, Romney SL. Preeclampsia and antioxidant nutrients: Decreased plasma levels of reduced ascorbic acid, a-tocopherol, and beta-carotene in women with preeclampsia. Am J Obstet Gynecol 1994; 171: 150-7. [CrossRef]

8. Roberts JM, Taylor RN, Musci TJ, Rodgers GM, Hubel CA, McLaughlin MK. Preeclampsia: An endothelial cell disorder. Am J Obstet Gynecol 1989; 161: 1200-4. [CrossRef]

9. Wang Y, Walsh SW, Guo J, Zhang J. The imbalance between thromboxane and prostacyclin in preeclampsia is associated with an imbalance between lipid peroxides and vitamin E in maternal blood. Am J Obstet Gynecol 1991; 165: 1695-700. [CrossRef] 
10. Bertsias G, loannidis JP, Boletis J, Bombardieri S, Cervera R, Dostal $C$, et al. EULAR recommendations for the management of systemic lupus erythematosus. Report of a Task Force of the EULAR Standing Committee for International Clinical Studies Including Therapeutics. Ann Rheum Dis 2008; 67: 195-205. [CrossRef]
11. Mills JA. Systemic lupus erythematosus. N Engl J Med 1994; 26: 1871-9. [CrossRef]

12. Magil AB, Puterman ML, Ballon HS, Chan V, Lirenman DS, Rae A, et al. Prognostic factors in diffuse proliferative lupus glomerulonephritis. Kidney Int 1988; 34: 511-7. [CrossRef] 